\title{
Multimodality molecular imaging of the alveolar-capillary barrier in lung disease using albumin based optical and PET tracers
}

\author{
Andrei Molotkov' ${ }^{1}$ Nikunj Bhatt', Mikhail Doubrovin', John Castrillon' ${ }^{1}$, Christopher Massa², Adam Gerber ${ }^{2}$, \\ Jeanine D'Armiento ${ }^{2}$, Monica Goldklang ${ }^{2}$ and Akiva Mintz ${ }^{1 *}$
}

\begin{abstract}
Inflammatory changes caused by viruses, bacteria, exposure to toxins, commonly used drugs and even surgical intervention have the potential of causing abnormal epithelial permeability, which is manifest as infiltrative processes on computed tomography (CT), including the widespread infiltrates seen in COVID-19 pneumonia and acute respiratory distress syndrome (ARDS). We utilized a previously published mouse model of ARDS, intranasal delivery of LPS, to induce the alveolar-capillary barrier permeability seen in lung disease. We intravenously injected mice with Cy7 or 68-Gallium ( ${ }^{68} \mathrm{Ga}$ ) labeled mouse albumin and imaged using optical imaging (OI)/CT and PET. We observed significantly increased lung levels of Cy7-albumin on 3D OI/CT, which matched the abnormal appearance on microCT. This uptake correlated with fluorescence seen on sectioned lungs. To examine the translational potential of these findings, we radiolabeled albumin with ${ }^{68} \mathrm{Ga}$. We found that in mice with LPS-induced lung injury, ${ }^{68} \mathrm{Ga}$-albumin PET correlated with our optical imaging findings and demonstrated abnormal activity in the lung fields, indicative of abnormal epithelial permeability. These findings indicate ${ }^{68} \mathrm{Ga}$-albumin can be utilized as a sensitive translational radiotracer for quantifying the abnormal epithelial permeability that is seen in various lung pathologies, including COVID-19 induced pneumonia and ARDS. The ability to use Cy7-albumin 3D OI/CT imaging as a preclinical translational surrogate for ${ }^{68} \mathrm{Ga}$-albumin offers an accessible high throughput means to rapidly screen potential therapeutics against lung diseases that clinically manifest with endothelial permeability.
\end{abstract}

Keywords: PET, ${ }^{68}$ Ga-albumin, ARDS, COVID

\section{Introduction}

Under normal circumstances, the alveolar-capillary membrane prevents fluid from entering the alveoli and hindering gas exchange. The alveolar-capillary membrane (or barrier) is composed of surface epithelial cells of the alveolar wall (type 1 pneumocytes), the endothelial cells of the capillaries and the basement membrane between them. Various diseases result in increased permeability in the

\footnotetext{
* Correspondence: am4754@cumc.columbia.edu

'Department of Radiology, Columbia University Irving Medical Center, 722 W. 168th St., New York, NY 10032, USA

Full list of author information is available at the end of the article
}

lungs that results in fluid filling the alveoli and preventing gas exchange. Acute lung injury (ALI) and acute respiratory distressed syndrome (ARDS) are caused by overproduction of cytokines, increased vascular permeability, protein-rich edema, and are leading causes of mortality in the patients [1]. Other causes of increased alveolarcapillary permeability in the lung include Pneumonitis and Pneumonia. Pneumonitis is an inflammation of lung parenchyma that is caused by a variety of airborne irritants. Pneumonia is pneumonitis triggered by bacterial, viral or fungal infections. Viral pneumonia with associated ARDS has emerged as a significant public health threat 
during the COVID-19 epidemic, with a significant minority of diagnosed patients suffering from lung infection and hospitalization [2]. Many of these patients end up requiring ventilators to achieve proper oxygenation. Of the patients placed on ventilators, $\sim 60 \%$ succumb to the virus [3]. While many patients who suffer COVID-related lung disease have comorbidities, it remains unpredictable which patients will suffer from lung complications until they demonstrate compromised oxygenation and abnormalities on plain chest radiography or CT. In addition to viral pneumonia, bacterial pneumonia [4] is one of the leading causes of hospitalization and morbidity worldwide with a reported age-standardized death rate of 41.7/ 100000 [5]. Hospital-acquired pneumonia significantly complicates treatment and negatively affects clinical prognosis and patient recovery. Computer tomography (CT) and plain chest radiography are the most often used standard method of diagnosing increased vascular permeability in the lungs, which manifests as lung infiltrates [6]. However, the presence of infiltrates indicates the late effect of the disease process, which is significant fluid in the lungs, and may not be a real-time measure of triaging early disease, quantifying the dynamics of the underlying alveolar and airway epithelial permeability, or an early predictor of response to treatment.

Lipopolysaccharide (LPS) induced lung injury is a commonly used model for ARDS, mimicking the inflammatory response observed in ARDS patients [7]. Local tracheal inhalation of LPS triggers robust migration of neutrophils into the lung parenchyma by $72 \mathrm{~h}$ after the exposure [8] followed by secondary fibrosis. Molecular mechanisms of the cellular response to LPS involve LPS binding to toll-like receptor 4 (TLR-4) followed by activation of the intracellular signaling kinases ERK1 and p38 and expression of the proinflammatory cytokines TNF $\alpha$ and IL-8 [9].

Albumin is an abundant constituent serum protein (35$50 \mathrm{~g} / \mathrm{l}$ ) with a molecular mass of $66.5 \mathrm{kDa}$. Under normal conditions, albumin cannot penetrate the endothelium and stays in circulation. However, during inflammation, endothelial permeability increases, and albumin exits blood vessels and can be found in damaged tissues.

We hypothesize that the real-time distribution of labeled albumin in the lungs is a feasible and informative method of directly evaluating the abnormal alveolar-capillary permeability that results in lung infiltrates. In this study, we evaluated the feasibility of ${ }^{68} \mathrm{Ga}$-albumin PET/CT to directly visualize the increased alveolar and airway epithelium permeability caused by inflammatory processes in the lungs.

\section{Results}

Cy7-albumin accumulates in lungs parenchyma after LPSinduced ARDS

We hypothesized that systemically injected labeled albumin will leak through the permeabilized alveolar- capillary membrane and allow sensitive real-time visualization of the disease process. To test this hypothesis, we first labeled recombinant mouse albumin with $\mathrm{Cy} 7$, a near infrared (NIR) dye, and injected it intravenously into mice $72 \mathrm{~h}$ after either intranasal LPS or intranasal PBS (control) treatment. Intranasal LPS treatment induces lung damage followed by formation of proteinrich edema and is thus used as a model of ARDS. Twenty-four hours after Cy7-albumin injection, we imaged mice for Cy7-albumin accumulation in the lungs using 3D optical imaging (OI)/CT. We found Cy7albumin uptake in the lungs of LPS treated mice $(n=5)$ corresponding to infiltrates on microCT, in contrast to controls (PBS treated, $n=4$ ) (Fig. 1a). To confirm the $\mathrm{OI} / \mathrm{CT}$ results, we euthanized the mice after imaging, and dissected, fixed and imaged the lungs using epifluorescent whole lung imaging. Similar to what we observed with OI/CT in live animals, we confirmed substantial $(p<0.01)$ Cy7-albumin accumulation in the lung parenchyma of the LPS-treated lungs $(n=5)$, in contrast to the PBS controls $(n=4)$ (Fig. 1b).

To confirm OI/CT images and demonstrate that the Cy7-albumin correlates with the LPS-induced lung damage, we sectioned the lungs after imaging and compared the $\mathrm{Cy} 7$ fluorescence in LPS-treated lungs versus controls (PBS). We found that in LPS-treated mice, there was lung parenchymal accumulation of Cy7-albumin, corresponding to swelling of the parenchyma and hemorrhagic areas seen after H\&E staining (Fig. 2). In contrast, in PBS-treated controls, lung morphology was unremarkable and Cy7-albumin was confined to intravascular spaces without significant accumulation in the lung parenchyma (Fig. 2).

\section{${ }^{68} \mathrm{Ga}$-albumin does not accumulate in normal lung parenchyma}

To examine if radiolabeled albumin is a feasible translational tracer for imaging real-time alveolar-capillary permeability, we performed whole body dynamic microPET imaging of ${ }^{68} \mathrm{Ga}$-albumin in normal mice to evaluate its physiologic distribution in the lungs and its distribution over an extended period of time (Fig. 3). Mice $(n=4)$ were given a single $i v$ dose of ${ }^{68} \mathrm{Ga}$-albumin $(\sim 7.2 \mathrm{MBq} /$ mouse) and a $4 \mathrm{~h}$ dynamic PET scan was acquired (Fig. 3a). We found that ${ }^{68} \mathrm{Ga}$-albumin was retained in blood circulation, including in the heart and large blood vessels, but cleared by at least $50 \%$ at $2 \mathrm{~h}$ (Fig. 3b). As expected, most of the ${ }^{68} \mathrm{Ga}$-albumin accumulated in the liver (Fig. 3a, b), reaching about $20 \% \mathrm{ID} / \mathrm{g}$ by $240 \mathrm{~min}$. In addition to the liver, we detected some accumulation of ${ }^{68} \mathrm{Ga}$-albumin in the bladder. There was low uptake in peripheral tissues, including in the lung parenchyma of normal mice (Fig. 3a). These results were confirmed post-necropsy by measuring radioactivity in blood and 

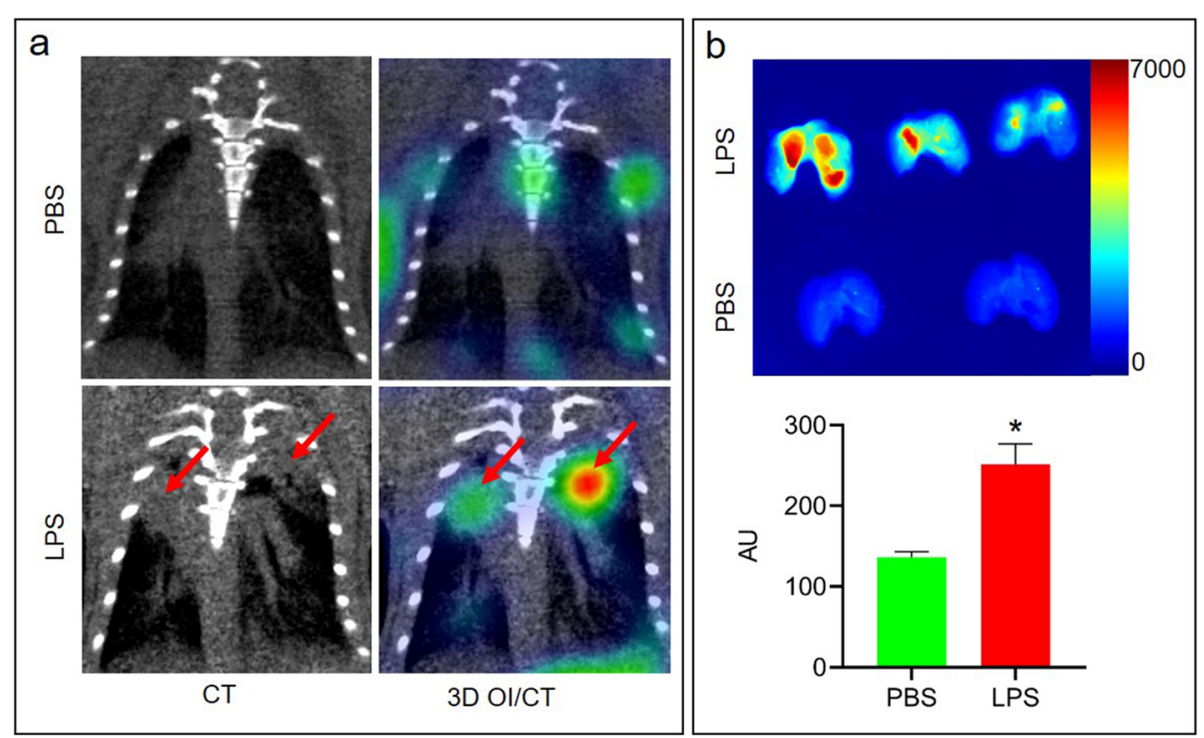

Fig. $13 \mathrm{D}$ OI/CT detection of LPS-induced lung inflammation using Cy7-albumin. a representative coronal images of Cy7-albumin uptake in the lungs of the PBS (top, $n=4$ ) and LPS (bottom, $n=5$ ) treated mice $24 \mathrm{~h}$ post injection. CT (left panels) and fusion 3D OI/CT (right panels) are shown. Note that infiltrates visible on CT (red arrows) are co-localized with Cy7-albumin accumulation detected by the 3D optical scan. b Cy7albumin is detected in dissected lungs of mice treated with LPS $(n=5)$ or PBS $(n=4$, control). *, $p<0.01$
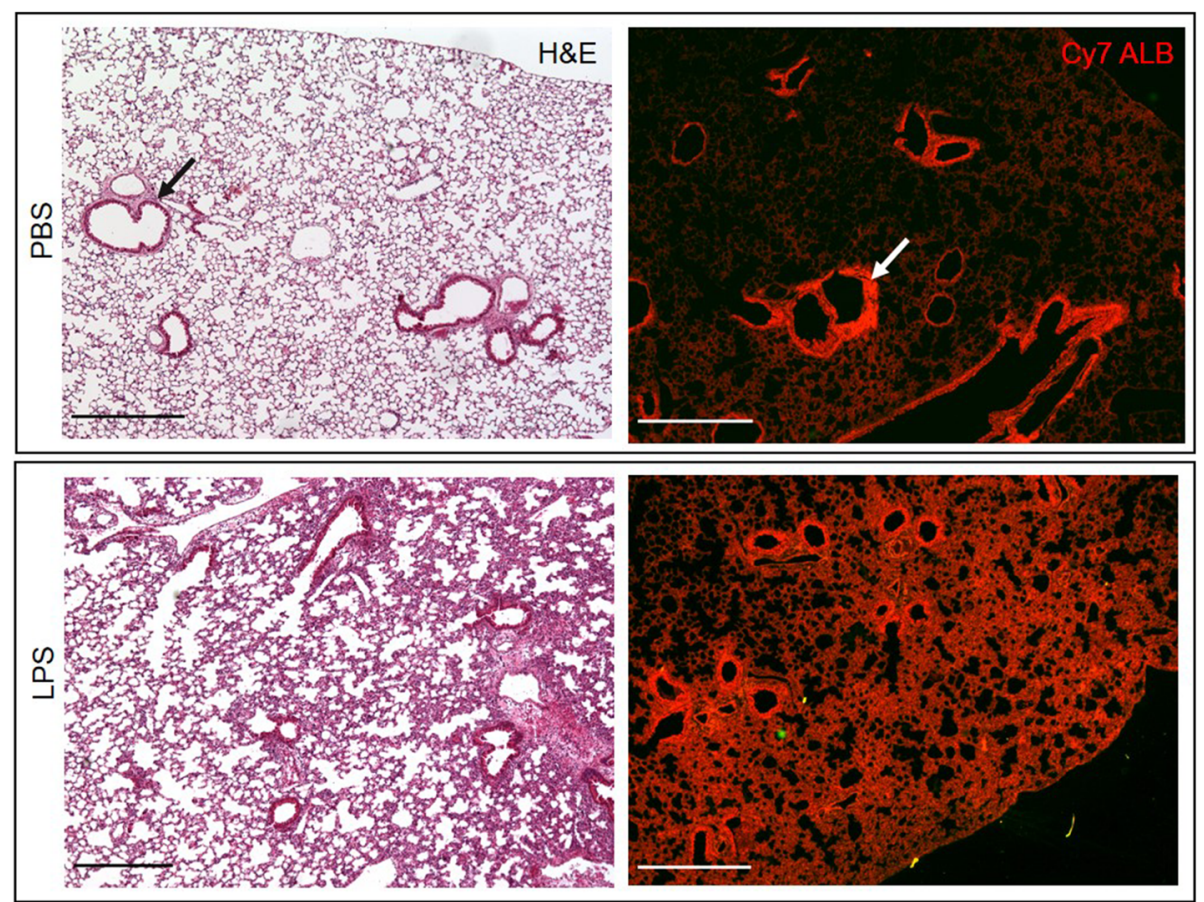

Fig. 2 Cy7-albumin uptake in lung parenchyma of mice $72 \mathrm{~h}$ after PBS or LPS treatment visualized under a fluorescent microscope. Bronchovascular bundles (arrows) are visualized on H\&E stained sections. Note considerable accumulation of Cy7-albumin (red) in parenchyma of LPS-damaged lungs $24 \mathrm{~h}$ post injection. Scale bars, $250 \mu \mathrm{m}$ 

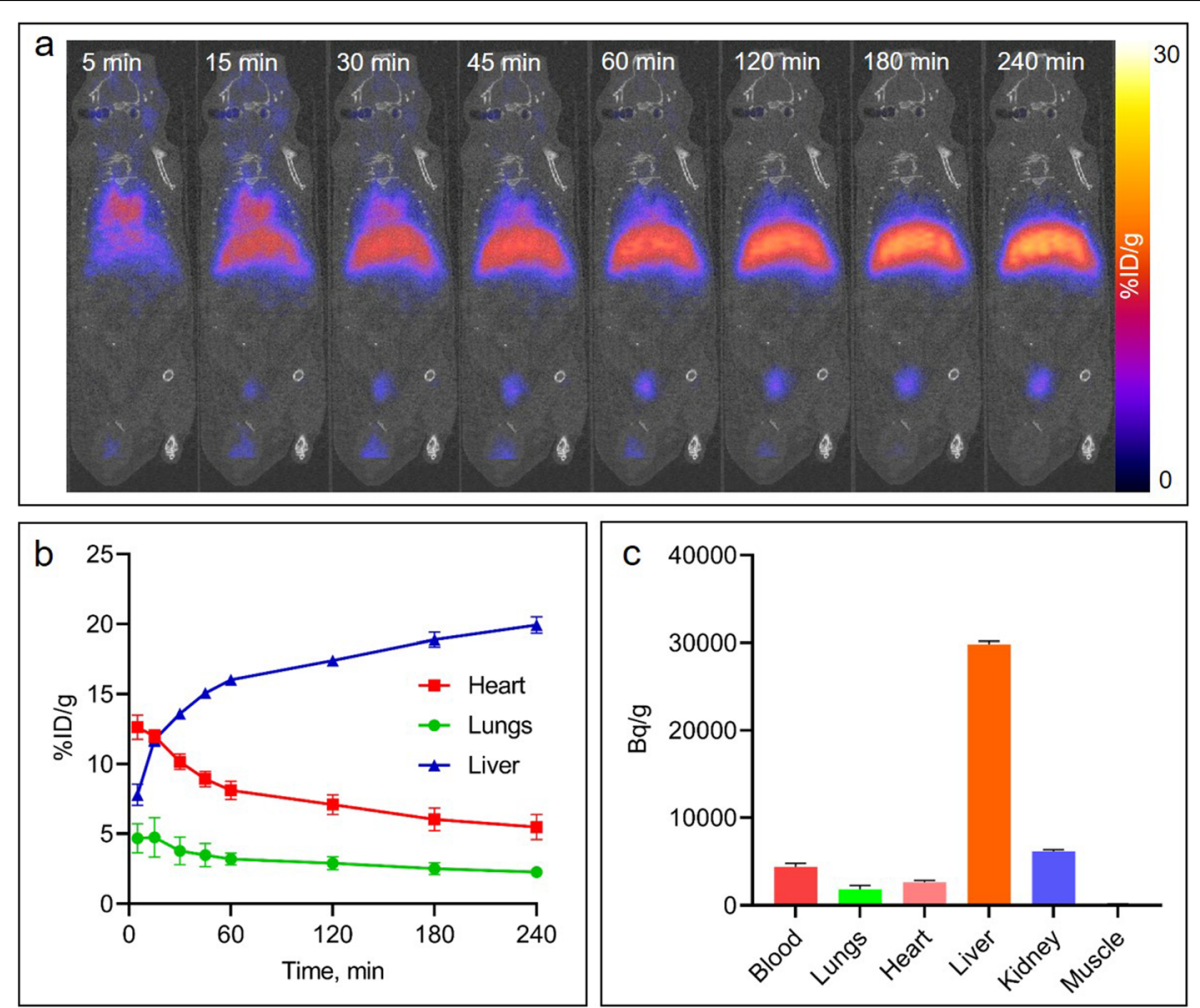

Fig. 3 Dynamic $4 \mathrm{~h}$ PET imaging after injection of ${ }^{68} \mathrm{Ga}$-albumin. a representative coronal images of a 240 min dynamic ${ }^{68} \mathrm{Ga}$-albumin PET scan in an untreated B6 mouse $(n=4)$. $\mathbf{b}$ dynamic ${ }^{68} \mathrm{Ga}$-albumin activity in the heart, liver and lungs of untreated B6 mice $(n=4)$. $\mathbf{c}$ biodistribution of ${ }^{68} \mathrm{Ga}$-albumin in tissues of untreated B6 mice $(n=4) \sim 4.5 \mathrm{~h}$ after injection

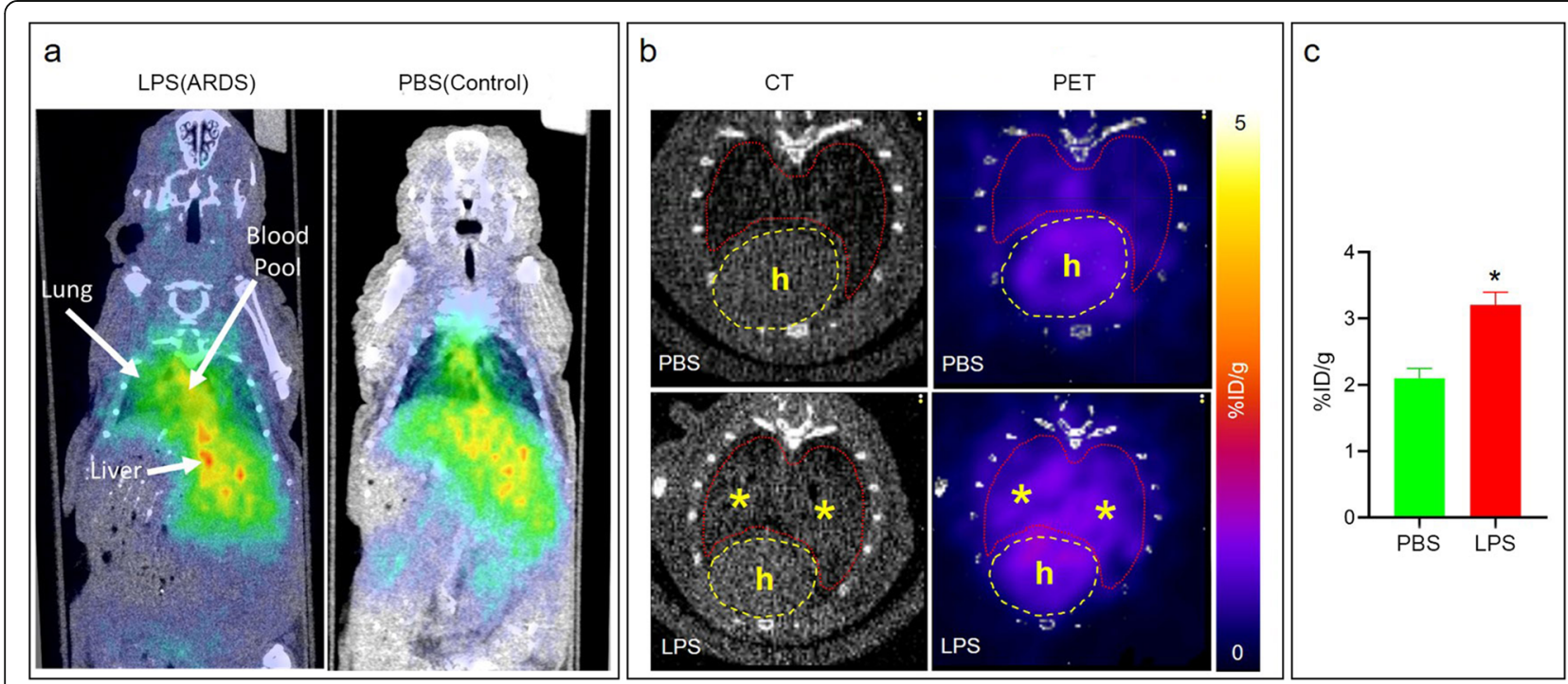

Fig. $4{ }^{68} \mathrm{Ga}$-albumin PET uptake in an LPS model of ARDS. a representative whole body coronal ${ }^{68} \mathrm{Ga}$-albumin PET/CT images $4 \mathrm{~h}$ after injection demonstrating significantly increased lung activity in LPS treated lungs compared to control, which only demonstrated physiologic uptake in the blood pool (heart) and liver. $\mathbf{b}$ quantification of ${ }^{68} \mathrm{Ga}$-albumin lung uptake on PET/CT axial images was derived by drawing ROIs on the CT image $\left(^{*}\right)$ and calculating the \% ID/g from the corresponding PET scan. $\mathbf{c}^{68} \mathrm{Ga}$-albumin lung uptake calculated from PET/CT images (as shown in b). ROls from lungs were compared between mice treated with intranasal LPS $(n=4)$ and intranasal PBS $(n=3)$, demonstrating significantly higher uptake in lungs of mice treated with LPS. ${ }^{*}, p<0.01$ 
tissues from mice $4 \mathrm{~h}$ after ${ }^{68} \mathrm{Ga}$-albumin injection (Fig. 3c).

\section{${ }^{68}$ Ga-albumin PET/CT identifies areas of LPS-induced lung permeability}

Our results using Cy7-albumin demonstrated the potential of labeled albumin as a marker of alveolar-capillary permeability. We therefore tested the lung uptake of ${ }^{68} \mathrm{Ga}$ albumin using PET/CT in mice pre-treated with intranasal LPS versus intranasal PBS. Static 30 min PET scans were acquired $4 \mathrm{~h}$ after ${ }^{68} \mathrm{Ga}$-albumin injection followed by microCT for anatomical reference (Fig. 4). We chose $4 \mathrm{~h}$ as a balance between blood pool clearance (Fig. 3b) and the $68 \mathrm{~min}$ half-life of ${ }^{68} \mathrm{Ga}$. Bq/g: Becquerel / g.

We found significantly $(p<0.01)$ increased ${ }^{68} \mathrm{Ga}$-albumin PET signal in the lungs of LPS treated mice $(n=4)$ corresponding to infiltrates on CT, in contrast to controls (PBS-treated mice, $n=3$ ) (Fig. 4). To confirm the $\mathrm{PET} / \mathrm{CT}$ results, we euthanized the mice immediately after imaging, and imaged just the lungs using PET. Similar to the 3D OI/CT and PET/CT results, we observed significant $(p<0.01){ }^{68} \mathrm{Ga}$-albumin accumulation in the lungs of the LPS-treated mice, in contrast to the PBS controls (Fig. 5).

\section{Discussion}

We demonstrated accumulation of the both Cy7albumin and ${ }^{68} \mathrm{Ga}$-albumin in an LPS-lung model of ARDS corresponding to lung parenchymal infiltrates seen on CT. Furthermore, we demonstrate that ${ }^{68} \mathrm{Ga}$-albumin appreciably clears the blood pool after $2 \mathrm{~h}$, which is relevant to evaluating lungs adjacent to the heart and great vessels.

Albumin has long been used as a marker to track vascular integrity. For example, Evan's blue (EB) dye, which binds serum albumin, is routinely used to test vascular permeability, blood-brain barrier integrity and cardiac function [10-12], but information about barrier integrity is only available during post-mortem analysis. To evaluate our hypothesis, we first labeled albumin with $\mathrm{Cy} 7$ and used 3D OI/CT to evaluate its distribution in a mouse model of LPS-induced pneumonitis compared to controls.

LPS is predominantly composed of components of the outer membrane of Gram-negative bacteria and is a potent initiator of inflammation. LPS binds to the CD14/ TLR/MD2/LBP receptor complex in monocytes and macrophages to induce production and secretion of proinflammatory cytokines [13, 14]. Intranasal LPS administration is an animal model of lung injury displaying many key features of ARDS [1] such as pulmonary edema and leukocyte infiltration, which has become a significant public health concern during the COVID-19 pandemic. We demonstrated that intranasal LPS induced lung infiltrates on $\mathrm{CT}$ with corresponding accumulation of Cy7-albumin on 3D OI/CT (Figs. 1 and 2). These results show that 3D OI/CT can be used as a means of real-time imaging of disease induced alveolar-capillary permeability, which can be important in evaluating early treatment effects of new drugs being developed to reduce permeability or treat underlying diseases such as COVID-19-induced pneumonia and ARDS. Using optical imaging to longitudinally visualize real-time pneumonia is a very accessible preclinical modality and can thus play a role in screening compounds for the treatment of COVID-19 related lung disease.

Our promising Cy7-albumin 3D OI/CT results prompted us to see if we can develop a translational PET scan to visualize albumin distribution in diseases that have increased lung permeability, including ARDS, COVID-19 pneumonia, blood-borne sepsis, primary lung infection, gastric aspiration, barotrauma, transfusion associated acute lung injury and hydrostatic chronic heart failure associated pulmonary edema. A previous study in

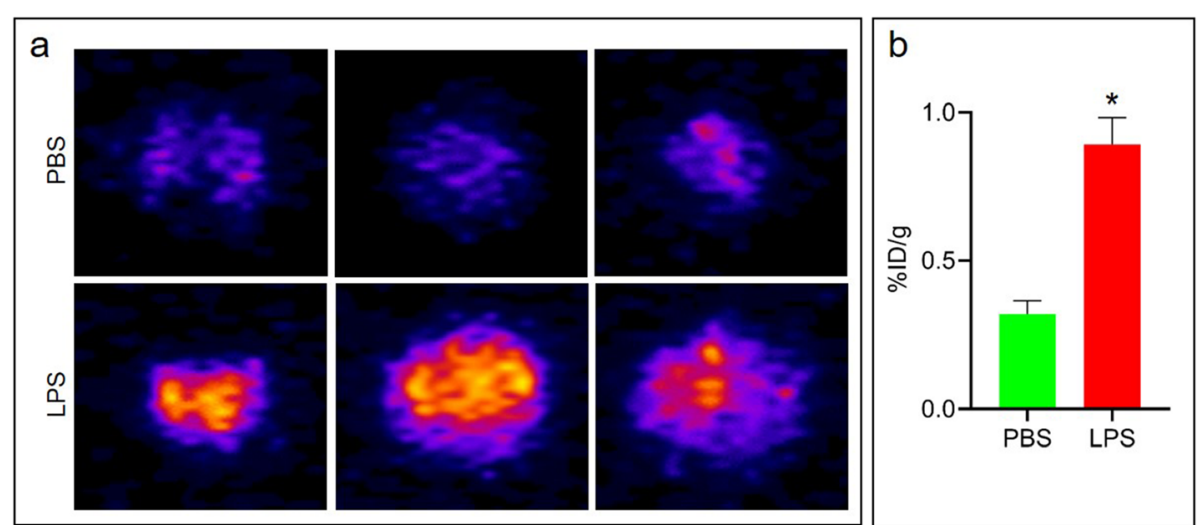

Fig. 5 a PET scans of dissected lungs from LPS $(n=4)$ and PBS $(n=3)$ treated mice $\sim 4.5 \mathrm{~h}$ after ${ }^{68} \mathrm{Ga}$-albumin injection. $\mathbf{b}$ quantification of uptake from (a). ${ }^{*} p<0.01$ 
humans already utilized albumin indirectly labeled with ${ }^{68} \mathrm{Ga}$-EB as a blood pool marker to differentiate liver hemangiomas, which showed increased activity, compared to focal hepatic lesions showing decreased activity [15]. Furthermore, the human study demonstrated very low ${ }^{68} \mathrm{Ga}$-EB-albumin uptake in the normal lungs [15], supporting our hypothesis that this might be a good approach to image damaged lungs. While their study demonstrated proof of concept using early imaging with albumin PET as a blood flow marker (30 min after injection), we were interested in imaging the albumin that leaked into the lungs after it was sufficiently cleared from the blood pool. We therefore performed whole body microPET imaging of normal mice for an extended period of time $(240 \mathrm{~min})$, significantly longer than the 30 min reported when albumin PET was used as a blood flow tracer. Furthermore, we directly labeled albumin using a NOTA bifunctional chelator to minimize any free ${ }^{68} \mathrm{Ga}$ from being generated over an extended period of time, as EB displays reversible binding to serum albumin. As expected, ${ }^{68} \mathrm{Ga}$-albumin initially remained in circulation while slowly accumulating in the liver during the first hour, with greater than 50\% cleared from circulation at $4 \mathrm{~h}$ (Fig. 3). We noted very low uptake of ${ }^{68} \mathrm{Ga}$ albumin in the peripheral tissues of normal mice (Fig. 3). Therefore, to minimize significant blood pool activity in the heart and great vessels that might interfere with adjacent lung, we chose to image at $4 \mathrm{~h}$ post injection.

As expected, intranasal LPS injection induced inflammation in the lung parenchyma (Fig. 2) followed by substantial accumulation of the ${ }^{68} \mathrm{Ga}$-albumin in the lungs. This was observed on PET scans of the mice and later confirmed in dissected lungs (Figs. 4 and 5). The differences between the LPS and control mice on PET images are underestimated in mice due to the ROI covering the entire lung, which is only a few $\mathrm{mm}$ away from the adjacent background liver and cardiac blood pool activity. The true difference is reflected in Fig. 5, where there is about a 3-fold increase in the LPS treated mice and controls. Due to the close proximity of the lungs, heart, and liver in mice, we could not completely avoid interference in the lower lungs from ${ }^{68} \mathrm{Ga}$ liver signal. While liver interference is more significant in mice due to the small size of their lungs, the vast majority of the lung is visible in much larger human lungs, as shown by Zhang et al. [15], although some interference at the lung bases will be inevitable.

Our end translational goal is to use PET imaging to quantitatively evaluate the effect of various experimental therapies on the alveolar-capillary barrier. To accomplish this in real time, it is necessary to use a short halflife radiotracer so we can image pre and post therapy. Furthermore, using ${ }^{68} \mathrm{Ga}$ significantly decreases the radiation exposure to the patient due to its comparatively short half-life when juxtaposed with ${ }^{64} \mathrm{Cu} \quad\left(\mathrm{T}_{1 / 2}=12 \mathrm{~h}\right)$ or ${ }^{89} \mathrm{Zr}\left(\mathrm{T}_{1 / 2}=78 \mathrm{~h}\right)$, other radiometals used in nuclear medicine. ${ }^{68} \mathrm{Ga}$ is available from a generator in many radiopharmacies that can be easily conjugated using a kit method, which significant eases distribution issues faced by cyclotron-based radiopharmaceuticals such as ${ }^{18} \mathrm{~F}$.

In Fig. 5. we demonstrated that at least 50\% of the albumin cleared the blood pool at $4 \mathrm{~h}$ post injection. This is a very conservative estimate because the ROI was taken to cover the whole lung, including areas adjacent to the liver, which overestimates the actual blood pool retention due to the high liver activity. This overestimation is clearly evident by visualizing the cardiac blood pool in the Fig. $5 \mathrm{a}$, which shows much greater clearance. Thus, we chose to test albumin based imaging due to its established use in humans as well as its ability to stay in the blood pool long enough to accumulate in diseased areas but not too long that it would remain in the blood pool at significant levels at the imaging time of $4 \mathrm{~h}$.

In conclusion, we report here optical or PET imaging of labeled albumin has the potential to detect the realtime lung permeability caused by inflammatory lung diseases or any condition that causes abnormal alveolarcapillary membrane permeability. We are therefore further investigating its use in disease models, such as COVID-19, for early diagnosis of lung disease, predication of disease severity, and for testing novel therapies prior to clinical trials.

\section{Material and methods \\ Animals}

C57BL/6NTac mice (B6, Taconic) were maintained on a normal mouse diet. All animal experiments were conducted according to protocols approved by the Institutional Animal Care and Use Committee of Columbia University Medical Center.

\section{Lipopolysaccharides treatment}

Under isoflurane anesthesia, mice were intranasally administered with $1 \mathrm{mg} / \mathrm{ml}$ of lipopolysaccharides (LPS) (MilliporeSigma, MA) solution in PBS at dose of $1.5 \mu \mathrm{l} / \mathrm{g}$. Control animals received equal volumes of intranasal PBS.

\section{Mouse albumin labeling with Cy7 and ${ }^{68} \mathrm{Ga}$}

Mouse serum albumin (ALB) (12-667, MilliporeSigma, MA) was labeled with Sulfo-Cyanine7 NHS ester (Cy7, near infrared die) according to manufacture instructions (Lumiprobe, MD) and purified using a PD10 column (Amersham Biosciences, NJ). Resulting Cy7-albumin (Cy7ALB) concentration was measured using a colorimetric assay and Cy7ALB was stored at $-70^{\circ} \mathrm{C}$ before use. To label ALB with ${ }^{68} \mathrm{Ga}$, NOTA-NCS $(0.42 \mathrm{mg})$ was added to a $1 \mathrm{ml}$ of $5 \mathrm{mg} / \mathrm{ml}$ solution of ALB in saline 
with $0.1 \mathrm{ml}$ of $0.1 \mathrm{M} \mathrm{Na}_{2} \mathrm{CO}_{3}$ (pH 9.0). The resulting solution was incubated for $30 \mathrm{~min}$ at $37^{\circ} \mathrm{C}$ using a thermomixer set at $600 \mathrm{rpm}$. After $30 \mathrm{~min}$, reaction mixture was loaded on preconditioned PD10 column (Amersham Biosciences, NJ) and washed with $1.35 \mathrm{ml}$ of saline. The eluate obtained was discarded and product (NOTAALB) was eluted with $2.2 \mathrm{ml}$ saline. ${ }^{68} \mathrm{Ga}$ was eluted from a ${ }^{68} \mathrm{Ge} /{ }^{68} \mathrm{Ga}$ generator (Eckert \& Ziegler, GalliaPharm, MA). Eluted ${ }^{68} \mathrm{Ga}(6-8 \mathrm{mCi}, 222-296 \mathrm{MBq})$ was equilibrated to $\mathrm{pH} 4.0$ with $1 \mathrm{M}$ sodium acetate buffer followed by addition of $50 \mu \mathrm{g}$ of NOTA-ALB. Reaction was incubated at $30^{\circ} \mathrm{C}$ for $15 \mathrm{~min}$ in a thermomixer (600 rpm). Formation of ${ }^{68} \mathrm{Ga}-\mathrm{NOTA}-\mathrm{ALB}$ was monitored by radio-TLC on ScanRAM 1a (LabLogic, FL) using Varian ITLC-SA strips (Varian, Inc., CA) and 50 mM EDTA (pH 5) as the mobile phase. Specific activity of ${ }^{68} \mathrm{Ga}-\mathrm{NOTA}$-ALB was $2850 \mathrm{mCi} / \mathrm{mole}(43 \mathrm{mCi} / \mathrm{g})$.

\section{D in vivo optical imaging and $\mathrm{CT}$}

To detect accumulation of $\mathrm{Cy} 7$ albumin in the lungs, control (PBS, $n=4)$ and LPS-treated $(n=5)$ B6 mice were injected $i v$ with $75 \mu \mathrm{l}$ of $\mathrm{Cy} 7$ labeled mouse albumin (Cy7ALB) $72 \mathrm{~h}$ after LPS/PBS treatment. 3D fluorescent images were acquired under isoflurane anesthesia using a 3D optical/CT scanner (MIlabs, Netherlands) 24 $\mathrm{h}$ after Cy7ALB administration. Immediately after 3D optical /CT scan, lungs were dissected, placed in $4 \%$ PFA in a 6-well plate, and imaged using the MILabs 3D optical scanner. Images were reconstructed using MILabs OI-PP version 1.8 software.

\section{PET experiments}

$72 \mathrm{~h}$ after intranasal LPS treatment, B6 mice were injected $i v$ with $436-473 \mu \mathrm{Ci}(16.1-17.5 \mathrm{MBq})$ of ${ }^{68} \mathrm{Ga}$ albumin. 4 to $5 \mathrm{~h}$ after ${ }^{68} \mathrm{Ga}$-albumin injection, mice were placed into a 4-mouse bed and $30 \mathrm{~min}$ static PET images were acquired using micro PET scanner (Siemens, Germany) followed by microCT (MIlabs, Netherlands) on the same bed for anatomical reference. Immediately after PET/CT acquisition, lungs were dissected, placed in 4\% PFA in a 6-well culture plate, and a $30 \mathrm{~min}$ PET static image of the isolated lungs was acquired. All PET images were reconstructed using 3DOSEM algorithm with 3 -iterations in $256 \times 256$ matrix (Inveon, Siemens, Germany) and analyzed using VivoQuant ver 4 (Invicro, MA).

\section{Biodistribution of ${ }^{68} \mathrm{Ga}$-albumin in mice}

B6 mice were injected $i v$ with a single dose of ${ }^{68} \mathrm{Ga}$-albu$\min (\sim 7.2 \mathrm{MBq} /$ mouse) and $4 \mathrm{~h}$ dynamic PET images were acquired using an Inveon microPET scanner (Siemens, Germany) followed by microCT (MILabs, Netherlands) on the same bed for anatomical reference. Immediately after $\mathrm{CT}$, mice were dissected and ${ }^{68} \mathrm{Ga}$ - albumin activity in the blood, heart, liver, kidney, and muscle was measured on a Hidex automatic gamma counter (Hidex, Finland).

\section{Cy7 epifluorescence of the dissected lungs}

Lungs were dissected immediately after optical 3D/CT scan and placed in 4\% PFA. Cy7 epi fluorescent images of dissected lungs were acquired using MILabs wholeanimal optical scanner (MIlabs, Netherlands) using a 20 s exposure time with a 657 excitation / 690 emission.

\section{Histology}

Lungs were fixed overnight in $4 \%$ PFA, embedded in paraffin and sectioned. Serial sections were used for $\mathrm{H} \& \mathrm{E}$ staining for morphological references. Cy7 fluorescence images were acquired on Nikon Eclipse Ti (Nikon, Japan).

\section{Quantification and statistical analysis}

Statistical analysis was performed using Prism 8.0. All data are represented as mean \pm standard error. Statistical $p$-values were calculated using two-tailed Student's t-test for unpaired samples.

\section{Abbreviations}

ALB: Mouse serum albumin; ALI: Acute lung injury; ARDS: Acute respiratory distress syndrome; $C T$ : Computer tomography; EB: Evan's blue;

LPS: Lipopolysaccharides; NIR: Near infrared fluorescence; Ol: Optical imaging; PET: Positron emission tomography; ROl: Region of interest; \%ID/g: Percent injected dose per gram

\section{Acknowledgments}

This publication was supported by the National Center for Advancing Translational Sciences (NCATS) UL1TR001873 (Reilly) through the TRx Accelerator Resource (Mintz).

\section{Consent to participate}

Not applicable.

\section{Code availability}

Not applicable.

\section{Authors' contributions}

All authors contributed substantially to this work. The author(s) read and approved the final manuscript.

\section{Funding}

This publication was supported by the National Center for Advancing Translational Sciences (NCATS) UL1TR001873 (Reilly) through the TRx Accelerator Resource (Mintz).

Availability of data and materials Not applicable.

\section{Ethics approval}

All animal experiments were conducted according to protocols approved by the Institutional Animal Care and Use Committee of Columbia University Medical Center.

Consent for publication

Not applicable. 


\section{Competing interests}

Authors declare no conflict of interests. A.M. serves as a paid constultant for Regeneron Pharmaceuticals.

\section{Author details}

1Department of Radiology, Columbia University Irving Medical Center, 722 W. 168th St., New York, NY 10032, USA. ${ }^{2}$ Center for LAM and Rare Lung Diseases, Department of Anesthesiology, Columbia University Medical Center, 722 W. 168th St., New York, NY 10032, USA.

\section{Received: 29 September 2020 Accepted: 20 November 2020}

Published online: 20 December 2020

\section{References}

1. Chen H, Bai C, Wang X. The value of the lipopolysaccharide-induced acute lung injury model in respiratory medicine. Expert Rev Respir Med. 2010;4(6): 773-83. https://doi.org/10.1586/ers.10.71.

2. Guan WJ, Ni ZY, Hu Y, Liang WH, Ou CQ, He JX, et al. Clinical characteristics of coronavirus disease 2019 in China. N Engl J Med. 2020. https://doi.org/10. 1056/NEJMoa2002032.

3. Zhou F, Yu T, Du R, Fan G, Liu Y, Liu Z, et al. Clinical course and risk factors for mortality of adult inpatients with COVID-19 in Wuhan, China: a retrospective cohort study. Lancet. 2020;395(10229):1054-62. https://doi.org/ 10.1016/S0140-6736(20)30566-3.

4. Prina E, Ranzani OT, Torres A. Community-acquired pneumonia. Lancet. 2015;386(9998):1097-108. https://doi.org/10.1016/s0140-6736(15)60733-4

5. Vos T, Barber R, Bell B, Bertozzi-Villa A, Biryukov S, Bolliger I, et al. Global, regional, and national incidence, prevalence, and years lived with disability for 301 acute and chronic diseases and injuries in 188 countries, 1990-2013: a systematic analysis for the Global Burden of Disease Study 2013. Lancet. 2015;386(9995):743-800. https://doi.org/10.1016/s0140-6736(15)60692-4.

6. Lanks CW, Musani Al, Hsia DW. Community-acquired pneumonia and hospital-acquired pneumonia. Med Clin North Am. 2019;103(3):487-501. https://doi.org/10.1016/j.mcna.2018.12.008.

7. Matute-Bello G, Frevert CW, Martin TR. Animal models of acute lung injury. Am J Physiol Lung Cell Mol Physiol. 2008;295(3):L379-99. https://doi.org/10. 1152/ajplung.00010.2008.

8. Bozinovski S, Jones JE, Vlahos R, Hamilton JA, Anderson GP. Granulocyte/ macrophage-colony-stimulating factor (GM-CSF) regulates lung innate immunity to lipopolysaccharide through Akt/Erk activation of NFkappa B and AP-1 in vivo. J Biol Chem. 2002;277(45):42808-14. https://doi.org/10. 1074/jbc.M207840200.

9. Medzhitov R. Recognition of microorganisms and activation of the immune response. Nature. 2007:449(7164):819-26. https://doi.org/10.1038/ nature06246.

10. Goldim MPS, Della Giustina A, Petronilho F. Using Evans Blue Dye to determine blood-brain barrier integrity in rodents. Curr Protoc Immunol. 2019:126(1):e83. https:/doi.org/10.1002/cpim.83.

11. Hopper J, Winkler AW, Elkinton JR. Simultaneous measurements of the blood volume in man and dog by means of Evans Blue Dye, T1824, and by means of carbon monoxide. II. Under abnormal conditions, including secondary shock. J Clin Invest. 1944;23(5):636-48. https://doi.org/10.1172/ jci101534.

12. Wolman M, Klatzo I, Chui E, Wilmes F, Nishimoto K, Fujiwara K, et al. Evaluation of the dye-protein tracers in pathophysiology of the blood-brain barrier. Acta Neuropathol. 1981;54(1):55-61. https://doi.org/10.1007/ bf00691332.

13. Kitchens RL. Role of CD14 in cellular recognition of bacterial lipopolysaccharides. Chem Immunol. 2000;74:61-82. https://doi.org/10.1159/ 000058750.

14. Dobrovolskaia MA, Vogel SN. Toll receptors, CD14, and macrophage activation and deactivation by LPS. Microbes Infect. 2002;4(9):903-14. https://doi.org/10.1016/s1286-4579(02)01613-1.

15. Zhang J, Lang L, Zhu Z, Li F, Niu G, Chen X. Clinical translation of an albumin-binding PET radiotracer 68Ga-NEB. J Nucl Med. 2015;56(10):1609_ 14. https://doi.org/10.2967/jnumed.115.159640.

\section{Publisher's Note}

Springer Nature remains neutral with regard to jurisdictional claims in published maps and institutional affiliations. 